\title{
Comparison of Doppler echocardiographic methods with heart catheterisation in assessing aortic valve area in 100 patients with aortic stenosis
}

\author{
J L Fischer, T Haberer, D Dickson, L Henselmann
}

II Medical

Department,

Cardiology and

Pneumology,

Städtisches

Krankenhaus

München-Neuperlach, Akademisches

Lehrkrankenhaus der Ludwig-MaximiliansUniversität München, Munich, Germany

J L Fischer

L Henselmann

III Medical

Department,

Cardiology,

Städtisches

Krankenhaus

München-Harlaching,

Munich

T Haberer

Biometric Centre for Therapeutic Studies,

Munich

D Dickson

Correspondence to:

Dr J L Fischer,

II Medizinisches Abteilung

Kardizinisches

Kardiologie und

Pneumologie, Städtisches

Krankenhaus München-
Neuperlach, Akademisches

Lehrkrankenhaus der

Ludwig-Maximilians-

Oniversität München,

Oskar-Maria-Graf-Ring 51,
81737 Munich, Germany.

Accepted for publication

4 October 1994

\begin{abstract}
Objective-To examine the practicability and accuracy of Doppler echocardiographic methods in determining aortic valve area.
\end{abstract}

Methods-Aortic valve areas determined by three methods using Doppler echocardiography (applying the continuity equation and the modified Gorlin formula using data from Doppler echocardiography and right heart catheterisation) were compared with values obtained by heart catheterisation.

Patients-100 consecutive patients with aortic stenosis aged between 34 and 83 years (mean (SD) $66(10)$ ).

Results-Differences in individual patients' measurements of aortic valve area by the three Doppler techniques varied by up to $0.56 \mathrm{~cm}^{2}$ compared with values obtained by heart catheterisation. On average, values obtained from Doppler echocardiographic methods lay up to $51 \%$ below and $78 \%$ above those obtained by heart catheterisation.

Conclusions-All three Doppler echocardiographic methods were practicable in routine clinical practice for patients of all ages, but they were of limited accuracy when compared with the aortic valve areas found invasively using the invasive Gorlin equation. However, these deviations may not always be due to inadequacies of the Doppler methods: they could also be caused by limitations in the Gorlin formula. Doppler methods can be repeated if required, they allow examination of the morphology of the valve, and they subject the patient to considerably fewer risks than the invasive procedure. An adequate strategy in determining the severity of aortic valve stenosis would be to calculate the valve area by Doppler echocardiography as well as considering the valvar aortic pressure gradient. The valve area alone should not be relied on exclusively, as has been the increasing practice in the past few years.

\section{(Br Heart f 1995;73:293-298)}

Keywords: aortic valve area; Doppler echocardiography; heart catheterisation; aortic stenosis
Doppler echocardiography enables the quantitative non-invasive determination of the severity of aortic valve stenosis. This can be done by determining the pressure gradient at the aortic valve ${ }^{1-3}$ and also by direct calculation of the aortic valve area. ${ }^{4-7}$ Indirect ways of determining the aortic valve area such as the $t-80$ ms method, ${ }^{8-10}$ the ratio of acceleration time to ejection time, ${ }^{11}$ and the difference in ejection time ${ }^{12}$ enable only a rough estimate of the severity of aortic stenosis. We applied a completely non-invasive method using the continuity equation ${ }^{6}$ and semi-invasive methods using the modified Gorlin formula $^{57}$ and compared these estimates of aortic valve area with the results obtained from invasive left heart catheterisation.

\section{Patients and methods}

One hundred and thirteen patients with suspected aortic valve disease were consecutively examined by $M$ mode echocardiography, cross sectional echocardiography, pulsed and continuous Doppler echocardiography, and heart catheterisation between May 1987 and January 1991. Six patients were not suitable for the study because they had grade III or IV aortic insufficiency on heart catheterisation ${ }^{13}$; one of these patients also showed stenosis of the aortic isthmus. Although all 113 patients had a gradient across the aortic valve, no satisfactory Doppler echocardiographic views could be obtained in two patients and retrograde passing of the calcified aortic valve was not possible during heart catheterisation in one patient. Aortic stenosis was diagnosed in one patient using the Bernoulli equation ${ }^{3}$ after performing Doppler echocardiography at a maximal flow velocity of $2 \cdot 1 \mathrm{~m} / \mathrm{s}$, but we found no peak to peak gradient on heart catheterisation. As a result of this, patients with a flow velocity of $2.1 \mathrm{~m} / \mathrm{s}$ or less were not included in the study (two further patients). One patient had auscultatory and echocardiographic and Doppler echocardiographic valvar aortic stenosis with a maximal flow velocity of $4.25 \mathrm{~m} / \mathrm{s}$ over the aortic valve but no peak to peak gradient during heart catheterisation. This remained unexplained. Thus 100 patients (46 women) were considered in this analysis.

Eighty three patients had sinus rhythm, 16 patients had atrial fibrillation, and one patient 
had alternating ventricular pacemaker and sinus rhythm. The mean age of the patients was 66 (SD 10) years (range 34-83 years). Heart catheterisation showed that 13 patients had pure aortic stenosis and 87 had aortic valve disease with clearly predominant aortic stenosis. Five patients also had mitral valve disease, one patient had pure mitral stenosis, and 33 patients had predominantly mild mitral insufficiency. One patient had an aneurysm of the ascending aorta. Coronary heart disease with over $50 \%$ stenosis was present in 37 patients, of whom 23 had one diseased coronary vessel, four two diseased vessels, and 10 three diseased vessels.

M MODE AND CROSS SECTIONAL ECHOCARDIOGRAPHY

An electronic sector scanner was used (Toshiba SSH-40A, SSH-60A, SSH-65A, and SSH-160A; 3.75 and $2.5 \mathrm{MHz}$ transducers) for the echocardiographic examinations. Left ventricular end systolic and end diastolic diameter, fractional shortening, left atrial diameter, and aortic valve opening were measured in $\mathbf{M}$ mode. The aortic outflow tract diameter was measured just below the valve by cross sectional echocardiography in the parasternal long axis view. The cross section of this diameter was determined to enable calculation of the valve area with the continuity equation (method I below). The mean of three measurements was used.

\section{DOPPLER ECHOCARDIOGRAPHY}

The Doppler echocardiography was performed using pulsed and continuous wave Doppler (Toshiba SDS 21B, SDS-60A, SDS$65 \mathrm{~A}$, and SDS-160A; $2.5 \mathrm{MHz}$ transducer). The continuous wave Doppler measurements were made by dedicated (pencil) and combined imaging transducers. The spectral curves were displayed on a Toshiba line scan recorder and a time motion hard copy printer. The recording speed was set to 50 $\mathrm{mm} / \mathrm{s}$ at the apparatus but ranged from $42-64$ $\mathrm{mm} / \mathrm{s}$. Doppler echocardiography was generally performed 24 hours before the heart catheterisation but in a few cases 48 hours before or after heart catheterisation. The prestenotic blood flow velocity, $\mathrm{v}_{1}$, was measured by pulsed wave Doppler mainly in the apical two chamber view (occasionally five chamber view) roughly $1 \mathrm{~cm}$ in front of the aortic valve. The transvalvar maximal flow velocity, $v_{2}$, was measured by continuous wave Doppler in all the usual views-that is, in apical two- and five-chamber views, parasternal right and left, and the suprasternal notch-and the clearest Doppler signal with the highest amplitude was used. In patients with atrial fibrillation the mean of five consecutive beats was used for $v_{1}$ and $v_{2}$. The ventricular ejection time and systolic ejection time were determined from the Doppler spectral curve $\mathrm{v}_{2} ; \mathrm{v}_{2}$ was planimetered three times by hand to obtain $\mathrm{v}_{\text {mean }}$. For the calculations using methods II and III, the cardiac output and stroke volume were calculated from right heart catheterisation by
Fick's method for 99 patients and by thermodilution for one patient. Aortic valve area $\left(\mathrm{cm}^{2}\right)$ was calculated from the following equations:

- Method $\mathrm{I}^{6}:$ aortic valve area $=\frac{\mathrm{v}_{1} \times(\mathrm{d} / 2)^{2} \times \pi}{\mathrm{v}_{2}}$

- Method II ${ }^{5}$ aortic valve area $=\frac{S V}{0.88 \times v_{2} \times V E T}$

Method III ${ }^{7}$ aortic valve area $=\frac{\mathrm{CO}}{\operatorname{SEP} \times \mathrm{v}_{\text {mean }}}$

where $v_{1}$ is prestenotic blood flow $(\mathrm{m} / \mathrm{s}), v_{2}$ transvalvar blood flow $(\mathrm{m} / \mathrm{s})$, d diameter of the aortic outflow tract $(\mathrm{cm}), \mathrm{SV}$, stroke volume $(\mathrm{ml}), \mathrm{v}_{\text {mean }}$, average planimetered blood flow velocity $(\mathrm{m} / \mathrm{s})$, CO cardiac output $(\mathrm{l} / \mathrm{min})$, VET ventricular ejection time (s), and SEP systolic ejection period ( $\mathrm{s} / \mathrm{min})$.

\section{HEART CATHETERISATION}

Right and left heart catheterisation was performed in 100 patients with valvar stenosis. Cardiac output was determined by Fick's method using the arteriovenous oxygen difference (between the ascending aorta and the pulmonary artery) and oxygen consumption tables. ${ }^{14}$ Cardiac output was also calculated by thermodilution. Catheterisation was performed using the retrograde femoral artery technique in all 100 patients. Using the consecutive pressure curves and at a speed of 100 $\mathrm{mm} / \mathrm{s}$ the mean systolic pressure gradient was determined planimetrically by hand from 3-5 beats, whereby planimetry was performed twice for each beat. The aortic valve area was calculated according to Gorlin and Gorlin and was again averaged from $3-5$ beats. ${ }^{15}$ In the pullback the mean planimetered systolic gradient was used for the Gorlin invasive formula. In all cases ventriculography (right anterior oblique projection $30^{\circ}, 30-45 \mathrm{ml}$ Ultravist contrast medium, flow $8-14 \mathrm{ml} / \mathrm{s}$ ) and aortography (left anterior oblique projection, $40-50 \mathrm{ml}$ Ultravist contrast medium, flow $14-16 \mathrm{ml} / \mathrm{s}$ ) were performed. The ejection fraction was calculated from ventriculograms by using the formula of Dodge et al. ${ }^{16}$ Coronary angiography was then performed.

\section{STATISTICAL METHODS}

Comparison of the aortic valve area obtained using each of the three methods with that obtained by heart catheterisation was investigated using the differences between the methods of measurement, which were described using mean differences and standard deviations. Limits of agreement and precision of bias were calculated using $95 \%$ confidence intervals for individual differences and for mean differences respectively. ${ }^{17}$

\section{Results}

One hundred patients with aortic stenosis were evaluable for the analysis of aortic valve area. The transvalvar maximal blood flow velocity at the aortic valve, $v_{2}$, was measured by continuous Doppler in the following positions: apical two chamber view (45 patients), apical five chamber view (11 patients), pencil 
Table 1 Descriptive summary of parameters measured in study population

\begin{tabular}{|c|c|c|c|}
\hline Parameter & $\begin{array}{l}\text { No of } \\
\text { patients }\end{array}$ & Mean (SD) & Range \\
\hline \multicolumn{4}{|l|}{ Transvalvar Doppler gradient at aortic valve } \\
\hline Mean pressure Doppler gradient $(\mathrm{mm} \mathrm{Hg})$ & 100 & $35(17)$ & $8-80$ \\
\hline Transvalvar blood flow $(\mathrm{m} / \mathrm{s})$ & 100 & $3.87(0.92)$ & $2 \cdot 18-5 \cdot 97$ \\
\hline Average planimetered blood flow velocity $(\mathrm{m} / \mathrm{s})$ & 100 & $2.84(0.76)$ & $1 \cdot 42-4 \cdot 50$ \\
\hline Prestenotic blood flow $(\mathrm{m} / \mathrm{s})$ & 100 & $0.83(0.19)$ & $0.44-1.45$ \\
\hline Ventricular ejection time (s) & 100 & $0.302(0.04)$ & $0 \cdot 196-0.392$ \\
\hline Systolic ejection period (s/min) & 100 & $23 \cdot 3(3 \cdot 8)$ & $15 \cdot 3-33 \cdot 3$ \\
\hline Diameter of left ventricular outflow tract $(\mathrm{cm})$ & 100 & $1.94(0.35)$ & $1 \cdot 0-2 \cdot 9$ \\
\hline Fractional shortening $(\%)$ & 84 & $34(13)$ & $10-86$ \\
\hline \multicolumn{4}{|l|}{ Average planimetered systolic presure gradient } \\
\hline$(\mathrm{mm} \mathrm{Hg})$ & 100 & $48(24)$ & $11-114$ \\
\hline Ejection fraction (\%) & 98 & $63(16)$ & $19-89$ \\
\hline Cardiac output (1/min): Fick method & 99 & $4.47(1.43)$ & $1 \cdot 7-8 \cdot 1$ \\
\hline Thermodilution & 98 & $4.59(1.56)$ & $1 \cdot 3-9 \cdot 4$ \\
\hline Stroke volume $(\mathrm{ml})$ & 100 & $61(23)$ & $23-125$ \\
\hline Heart rate (beats/min) & 100 & $76(13)$ & $51-117$ \\
\hline Aortic valve area $\left(\mathrm{cm}^{2}\right)^{\star}$ & 100 & $0.77(0.43)$ & $0 \cdot 21-2 \cdot 22$ \\
\hline
\end{tabular}

^On heart catheterisation. apical (3 patients), right parasternal (14 patients), left parasternal (1 patient), suprasternal (1 patient), apical two and five chamber view (11 patients), apical two and five chamber view and right parasternal (12 patients), apical two and five chamber and right parasternal and suprasternal (two patients). Thus measurements of blood flow velocity in the apical view were obtained in 84 patients. Table 1 shows the values of cardiac parameters for the study population.

To measure the agreement (or rather, discrepancies) between the resulting aortic valve areas, we calculated the differences (measurement errors) between the aortic valve area obtained by heart catheterisation and that obtained by the three Doppler methods. From table 2, the limits of agreement (95\% confidence intervals for the differences) show, for individual patients, how far the value of aortic valve area obtained by each method is likely (with $95 \%$ probability) to lie from the value obtained by heart catheterisation. For example, the estimate obtained by method III may be up to $0.48 \mathrm{~cm}^{2}$ below and $0.47 \mathrm{~cm}^{2}$ above the value obtained by heart catheterisation. The precision of the bias $(95 \%$ confidence interval for the mean difference) shows to what extent each method is likely on average to underestimate or overestimate the aortic valve area. For example, method I is likely to underestimate (with $95 \%$ probability) the aortic valve area obtained by heart catheterisation by 0.02 to $0.10 \mathrm{~cm} .{ }^{2}$ Method II is also likely to underestimate on average and method III may overestimate or underestimate on average. The widths of the $95 \%$ confidence intervals are similar for all methods $\left(0.87\right.$ to $0.95 \mathrm{~cm}^{2}$ for limits of agreement and 0.08 to $0.09 \mathrm{~cm}^{2}$ for precision of bias).

This analysis assumes the differences are constant over the whole possible range of values. As is seen in figures 1 to 3, there is a tendency for differences to increase with larger values of aortic valve area. This indicates that the limits of agreement in table 2 may be too wide for low values, too narrow for high values, and correct for aortic valve areas which lie close to the mean value (around $0.8 \mathrm{~cm}^{2}$ ).

This proportionality of the differences to the value of aortic valve area obtained by heart catheterisation can be removed by logarithmically transforming the data. The analysis was therefore repeated using logarithmically transformed data and the antilogged results are shown in table 3 . These are dimensionless ratios - for example, a value of less than 1.0 indicates that the area obtained by the Doppler method is smaller than that obtained by the invasive Gorlin method. The limits of agreement show that for individual patients method I is likely (with $95 \%$ confidence) to yield a value between $51 \%$ below and $75 \%$ above the aortic valve area obtained by heart catheterisation. Method II is likely to yield values between $49 \%$ below and $51 \%$ above and method III between $44 \%$ below and $78 \%$ above those values determined by heart catheterisation. The precision of bias shows that on average method I is likely to 
Table 2 Limits of agreement and precision of bias for three Doppler methods of measuring aortic valve area compared with heart catheterisation in patients with aortic stenosis.

Values are $\mathrm{cm}^{2}$

\begin{tabular}{llll}
\hline $\begin{array}{l}\text { Doppler } \\
\text { method }\end{array}$ & $\begin{array}{l}\text { Mean difference (SD) } \\
\text { in area by Doppler and } \\
\text { heart catheterisation }\end{array}$ & Limit of agreement * & Precision of biast \\
\hline I & $-0.06(0.22)$ & -0.50 to 0.38 & -0.10 to 0.02 \\
II & $-0.10(0.21)$ & -0.56 to 0.31 & -0.15 to 0.06 \\
III & $-0.006(0.24)$ & -0.48 to 0.47 & -0.05 to 0.04 \\
\hline
\end{tabular}

$\star 95 \%$ confidence interval for difference between the two methods, or how far value is likely to be with $95 \%$ probability from value by heart catheterisation. $\dagger 95 \%$ confidence interval for mean difference, or the average underestimation or overestimation of aortic valve area as measured by heart catheterisation.

Table 3 Limits of agreement and precision of bias for three Doppler methods of measuring aortic valve area compared with heart catheterisation in patients with aortic stenosis. Values are antilogs.

\begin{tabular}{llll}
\hline $\begin{array}{l}\text { Doppler } \\
\text { method }\end{array}$ & $\begin{array}{l}\text { Mean difference (SD) } \\
\text { in area by Doppler and } \\
\text { heart catheterisation }\end{array}$ & Limit of agreement & Precision of biast \\
\hline I & $0.92(1.38)$ & 0.49 to 1.75 & 0.86 to 0.98 \\
II & $0.88(1.32)$ & 0.51 to 1.51 & 0.82 to 0.92 \\
III & $0.99(1.35)$ & 0.56 to 1.78 & 0.93 to 1.05 \\
\hline
\end{tabular}

${ }^{\star} 95 \%$ confidence interval for difference between the two methods, or how far value is likely to be with $95 \%$ probability from value by heart catheterisation. $+95 \%$ confidence interval for mean difference, or the average underestimation or overestimation of aortic valve area as measured by heart catheterisation. underestimate aortic valve area by about $2-14 \%$ (with $95 \%$ probability) and method II by $8-18 \%$; method III may underestimate by up to $7 \%$ or overestimate by up to $5 \%$ on average.

\section{Discussion}

The assessment of the severity of an aortic stenosis without heart catheterisation is often difficult. Nowadays, non-invasive Doppler echocardiography estimates severity in addition to detecting such stenosis. Good agreement between such values and those obtained by invasive heart catheterisation in determining maximal aortic stenosis pressure gradients have been shown. ${ }^{18-24}$ Nevertheless, discrepancies have also been reported, and in particular it has been warned that the true maximal pressure gradient cannot be recorded using the Doppler echocardiographic methods..$^{25-27}$ As well as determining the gradient, Doppler echocardiography offers the possibility of measuring aortic valve area. ${ }^{28-35}$ This is particularly important since the determination of aortic valve area is indispensable in judging the severity of aortic valve stenosis in patients with reduced ventricular function. ${ }^{36-37}$

We were particularly interested in the applicability and accuracy of the Doppler echocardiographic methods in determining the aortic valve area. ${ }^{3638}$ All parameters required for the determination of aortic valve area could be derived for 100 of the 113 patients in this study. This indicates the practicability of Doppler echocardiographic methods in patients with aortic stenosis, as our patients were of varying ages and consecutively referred. Experience shows that measurement of the aortic outflow tract in calcified aortic stenosis is often associated with a measurement error. With methods II and III, the aortic valve area was obtained using data from Doppler echocardiography and right heart catheterisation-namely, the stroke volume in method II and the cardiac output in method III. Cardiac output and stroke volume could also have been obtained non-invasively by Doppler echocardiography. This is, however, extremely time consuming, requires considerable experience, ${ }^{39} 40$ and is often technically impossible in older patients.

To measure the disagreement between each of the Doppler echocardiographic methods and heart catheterisation, pairwise differences of aortic valve area were calculated for each of the 100 patients. The valve area obtained from the Doppler methods differed by up to $0.56 \mathrm{~cm}^{2}$ ( $95 \%$ confidence limit) from the value obtained by heart catheterisation. This must be considered as unacceptable, given that a value of $0.8 \mathrm{~cm}^{2}$ or less indicates aortic valve replacement and a larger value indicates a more conservative approach. Considering the disagreements as percentage differences, we found that the Doppler echocardiographic values were likely to lie somewhere between $51 \%$ below and $78 \%$ above those obtained by heart catheterisation (with $95 \%$ probability). Comparing the variability in individual patient measurements, we found that methods I and III overestimated or underestimated the aortic valve area to a similar extent. Method II had a slightly narrower confidence interval, but methods I and II largely produced similar results. Methods I and II are likely to underestimate the aortic valve area on average and method III may underestimate or overestimate aortic valve area.

The considerable discrepancies between results obtained by the three methods and those obtained by heart catheterisation require some explanation. Some recent evidence indicates that the Gorlin formula, the accepted standard for assessing aortic stenosis, has some accuracy limitations for aortic valve areas between $0.5 \mathrm{~cm}^{2}$ and $1.5 \mathrm{~cm}^{2} 273041$ and low flow states, for which the Gorlin equation may be less accurate than the Doppler derived aortic valve area estimated by the continuity equation. ${ }^{42}$ One important determination required by the Gorlin formula is an accurate measure of the pressure gradient. Several potential sources of error in cardiac catheterisation laboratories include the retrograde placement of a left ventricular pressure catheter across a stenotic aortic valve. This further reduces the effective orifice area, which may alter the gradient, especially in patients with aortic valve stenosis. Retrograde replacement can also induce aortic regurgitation and alter the gradient. Furthermore, the pressure gradient at the aortic valve may change when contrast medium is applied in the left ventricle or when the patient receives medication. There has been some discussion that the Gorlin constant is not a constant at all but an empirically derived estimate that almost certainly varies with transvalvar flow and pressure. ${ }^{41}$ The aortic valve area derived from this Gorlin 
formula may greatly underestimate the true orifice size, particularly in patients with low cardiac output. ${ }^{43}$

The continuity equation in method I offers several advantages over catheterisation. Unlike the Gorlin equation, the continuity equation does not require the use of an empirically derived constant or the calculation of flow rates, pressure gradients, heart rate, or systolic ejection period. In addition, the continuity equation does not require an invasive procedure, and therefore serial evaluation by Doppler echocardiography is made easier. The accuracy of the estimates of aortic valve area in the continuity equation has been shown to be dependent on the reliability of measurements of the diameter of and velocity in the left ventricular outflow tract. ${ }^{44}$ The measurement of the diameter is a potential source of error. A variability of $2 \mathrm{~mm}$ in the diameter of the left ventricular outflow tract diameter could result in an $18 \%$ error in the estimated aortic valve area. ${ }^{45} \mathrm{We}$ also emphasise that when measuring the prestenotic flow, $v_{1}$, the sample volume should be proximal to the aortic annulus to avoid accelerated velocity. ${ }^{6}$ Different values for the prestenotic flow can lead to differing results for the aortic valve area, especially when this area is around $1 \mathrm{~cm}^{2}{ }^{42}$ In our experience, flow in the left ventricular outflow tract should be mapped from the aortic valve into the left ventricle, where the prestenotic blood flow can then be seen. With experience varying values for prestenotic flow are no longer a problem.

Methods II and III are considered to be semi-invasive as they require data from invasive right heart catheterisation and those from non-invasive Doppler echocardiography. They have the advantage over the Gorlin formula that left heart catheterisation is not necessary. Serious complications can occur during left heart catheterisation-for example, as the result of dislodged calcium deposits at the aortic valve or the injection of contrast medium into the left ventricle. Unlike for the continuity equation the prestenotic flow velocity, $\mathrm{v}_{1}$, and the diameter of the left ventricular outflow tract are not required. Cardiac output and stroke volume are determined by right heart catheterisation and thus the transvalvar flow, which directly influences the equation, is determined exactly. Although the cardiac output used in methods II and III is the same as that used in the Gorlin invasive formula, the agreement of these methods was not better than that obtained by the continuity equation. The equation for method II also contains a constant and both methods II and III are frequency dependent.

The pairwise comparisons of values for all methods show the potential differences between Doppler echocardiographic methods and heart catheterisation in determining aortic valve area. We observed extreme deviations for individual measurements, such deviations increasing with increasing aortic valve area. Furthermore, the deviations observed may not always be due to inadequa- cies of the Doppler methods: they could also be caused by limitations in the Gorlin formula. Further similar studies on larger numbers of patients are required to achieve more precise estimates of differences between the methods.

We thus conclude and recommend that the valvar aortic gradient should always be determined when evaluating aortic valvar stenosis. This is crucial, especially for patients with normal ejection fraction, just as it is important to consider the aortic valve area in patients with impaired left ventricular function. We found that $8 \%$ of patients in our study whose aortic valve area indicated valve replacement according to the Doppler method had a low peak to peak gradient. Similarly, $8 \%$ of the patients whose aortic valve area indicated valve replacement according to the invasive Gorlin method had a low gradient. These two groups of patients were exclusive (no patient was common to both groups), and none of them had clinical symptoms of severe aortic stenosis. Invasive left heart catheterisation is not strictly necessary, even in elderly patients, because the Doppler echocardiographic technique is practicable and the limitations of the Gorlin formula apply to patients of all ages.

1 Agatson AS, Chengot M, Rao A, Hildner F, Samet P. Doppler diagnosis of valvular aortic stenosis in patien over 60 years of age. $A m \mathcal{F}$ Cardiol 1985;56:106-9.

2 Currie P, Steward J, Reeder G, et al. Continuous wave Doppler echocardiographic assessment of severity of calcified aortic stenosis: a simultaneous Dopplercatheter correlative study in 100 adult patients. Circulation 1985;71:1162-9.

3 Hatle L, Angelsen A, Tromsdal A. Noninvasive assessment of aortic stenosis by Doppler ultrasound. Br Heart F 1980;43:284-92.

4 Fujii K, Kitabatake A, Asao M, et al. Noninvasive evaluation of valvular stenosis by a quantitative Doppler technique. Fournal of Cardiovascular Ultrasonography 1984;3: nique.

5 Kosturakis D, Allen HD, Goldberg SI, Sahn DJ, ValdesCruz LM. Noninvasive quantification of stenotic valve areas by Doppler echocardiography. Am f Cardiol 1984; 3:1256-62.

6 Skjaerpe T, Hegrenaes L. Noninvasive estimation of valve area in patients with aortic stenosis by Doppler ultrasound and two-dimensional echocardiography. Circulation 1985;72:810-8.

7 Warth DC, Atewart WJ, Block PC, Weyman AE. A new method to calculate aortic valve area without left heart catheterisation. Circulation 1984;70:978-83.

8 Dennig K, Henneke KH, Rudolf W. Vergleich Dopplerechocardiographischer Methoden zur Bestimmung des Schweregrades von Aortenklappenstenosen. $Z$ Kardiol 1987;13 (suppl 1).

9 Henneke KH, Dennig K, Dacia S, Rudolph W. Vergleich Doppler-echocardiographischer Methoden zur Bestimmung der Öffnungsfläche von Aortenklappenstenosen. Z Kardiol 1990;63(suppl 2)

10 Ramirez ML, Noll HE, Wong M, Josephson MA, Shah PM. Doppler assessment of aortic valve area in aortic PM. Doppler assessment of aortic valve area in aortic
stenosis: use of t -80 sec velocity measurement. Circulation 1985;574(suppl III).

11 Curtius JM, Opgenorth R, Loogen F. Klinische Bedeutung der dopplerechokardiographischen Bestimmung des Schweregrades von Aortenklappenstenosen. $Z$ Kardiol 1987;76:269-75.

12 Zoghbi WA, Galan A, Quinones MA. Accurate assessment of aortic stenosis severity by Doppler echocardiography independent of aorticjet velocity. Am Heart $\mathrm{f}$ 1988;116:855-63.

13 Sellers RD, Levy MJ, Amplatz K, Lillehei CW. Left retrograde cardioangiography in acquired cardiac disease. Am 7 Cardiol 1964;14:437-47.

14 Boothey B, Berkson B, Dunn F. Normogramm zur Bestimmung des Grundumsatzes und der Normalitätswahrscheinlichkeit in Prozent. Am F Physiol 1936;116. 468 .

15 Gorlin R, Gorlin SG. Hydraulic formula for calculation of the area of the stenotic mitral valve, other cardiac valves 
and central circulatory shunts. Am Heart $f$ 1951;41: 109

16 Dodge HT, Sandler H, Ballew DW, Lord JD Jr. The use of bi-plan angiography for the measurement of left ventricular volume in man. Am Heart $\mathcal{F} 1960 ; 60: 762$.

17 Bland JM, Altman DG. Statistical methods for assessing agreement between two methods of clinical measurement. Lancet 1986;i:307-10.

18 Berger M, Berdoff RL, Gallerstein PE, Goldberg E. Evaluation of aortic stenosis by continuous wave Doppler ultrasound. $₹ \mathrm{Am}$ Coll Cardiol 1984;3:150-6.

19 Hatle L. Noninvasive assessment and differentiation of left ventricular outflow obstruction with Doppler ultrasound. Circulation 1981;64:381-7.

20 Hatle L. Aortic valve stenosis. INSERM symposium series. 1982;111:313-22.

21 Hatle L. Maximal blood flow velocities-hemodynamic data obtained noninvasively with Doppler. Ultrasound Med Biol 1984;10:225-37.

22 Hatle L. Assessment of aortic stenosis with Doppler ultrasound. Int $\mathcal{F}$ Cardiol 1985; 7:127-8.

23 Hegrenaes L, Hatle L. Aortic stenosis in adults. Noninvasive estimation of pressure differences by continuous wave Doppler echocardiography. Br Heart $f$ 1985;54:396-404.

24 Williams G, Labovitz A, Nelson J, Kennedy H. Value of multiple echocardiographic views in the evaluation of aortic stenosis in adults by continuous wave Doppler. Am $\mathcal{F}$ Cardiol 1985;55:445-9.

25 Holper D, Böttcher D, Baew-Chritow T, Adam W Pfannenstiel P. Kann die moderne nichtinvasive kardiolPfannenstiel P. Kann die moderne nichtinvasive kardiologische Diagnostik die Herzkatheteruntersuchung bei erworbenen Herzklappenfehl

26 Krafchek J, Robertson JH, Radford M, Adams D, Kisslo J. A reconsideration of Doppler assessed gradients in suspected aortic stenosis. Am Heart $\mathcal{F}$ 1985;110:765-73.

27 Stamm BR, Martin RP. Quantification of pressure gradients across stenotic valves by Doppler ultrasound. f Am Coll Cardiol 1983;4:707-18.

28 Ohlsson J, Wranne D. Noninvasive assessment of valve area in patients with aortic stenosis. $7 \mathrm{Am}$ Coll Cardio 1986;7:501-8.

29 Otto CM, Pearlman AS, Comess KA, Reamer RP, Janko CL, Huntsman IL Determination of the stenosic aortic valve area in adults using Doppler echocardiography. valve area in adults using Dopp
$f$ Coll Cardiol 1986;7:509-17.

30 Richards KL, Cannon SR, Miller JF, Crawford MH Calculation of aortic valve area by Doppler echocardiography: a direct application of the continuity equation raphy: a direct application

31 Seitz WS, Kashani IA. Non-invasive determination of the aortic valve area in stenosis: hydraulic orifice formula for application to echocardiography and correlation with catheterization. Eur Heart $\mathcal{F} 1983 ; 4: 31-40$.
32 Teirstein P, Yeager M, Yock PG, Popp RL. Doppler echocardiographic measurement of aortic valve area in aortic stenosis: a non-invasive application of the Gorlin formula. F Am Coll Cardiol 1986;8:1059-65.

33 Veyrat C, Gourtchiglouian C, Dumora P, et al. A new non-invasive estimation of the stenotic aortic valve area by pulsed Doppler mapping. Br Heart $\mathcal{f} 1987 ; 57: 44-50$.

34 Zhang Y, Myhre E, Nitter-Hauge S. Noninvasive quantification of the aortic valve area in aortic stenosis by

35 Zoghbi WA, Farmer KL, Soto JG, Nelson JG, Quinones MA. Accurate noninvasive quantification of stenotic valve area by Doppler echocardiography. Circulation valve area by
1986;73:452-9.

36 Fischer $\pi$, Haberer $T$, Lindlbauer $R$. Zuverlässige Operationsindikationsstellung mittels Dopplerecho-
kardiographie bei valvulären Aortenstenosen durch kardiographie bei valvulären Aortenstenosen durch Ermittlung des Aortenklappengradienten
nungsfäche. $Z$ Kardiol 1988;153(suppl 1)

37 Smith N, McAnulty JH, Rahimtoola SH. Severe aortic stenosis with impaired left ventricular function and clinical heart failure: results of valve replacement. Circulation 1978;58:255-64.

38 Fischer JL, Haberer T, Lindlbauer R. Vergleich Dopplerechokardiographischer Methoden zur Bestimmung der Aortenklappenöffnungsfläche von valvulären Aortenstenosen. Z Kardiol 1988;152(suppl 1).

39 Ihlen $\mathrm{H}$, Amlie J, Dale J, et al. Determination of cardiac output by Doppler echocardiography. Br Heart $f$ 1984; 51:54-60.

40 Schuster A, Nanda N. Doppler echocardiographic measurement of cardiac output: comparison with a nonsurement of cardiac output: comparison with

41 Cannon S, Richards K, Crawford M. Hydraulic estimation of stenoticorifice area: a correction of the Gorlin formula Circulation 1985:71:1170-8.

$42 \mathrm{Oh} J K$, Talciercio CP, Holmes DR, et al. Prediction of the severity of aortic stenosis by Doppler aortic valve area determination: prospective Doppler catheterization correlation in 100 patients. $\mathcal{F}$ Am Coll Cardiol 1988;11 $1227-34$.

43 Segal J, Lerner D, Miller C, Mitchell S, Alderman E, Popp $R$. When should Doppler determined valve are be better than Gorlin formula? Variation in hydraulic constants in low flow states. F Am Coll Cardiol 1987; 9:1294-305.

44 Grayburn P, Smith M, Harrison M, Gurley JC, De Maria AN. Pivotal role of aortic valve area calculation by the AN. Pivotal role of aortic valve area calculation by the continuous equation for Doppler assessment of aortic reguritation. $7 \mathrm{Am}$ Coll Cardiol 1988;61:376-81.

45 Oro MC. Simplification of the Doppler continuity equation for calculation of stenotic valve area. $f \mathrm{Am}$ Soc for calculation of stenotic
The 1995 Annual Meeting of the British Cardiac Society will take place at the Conference Centre, Harrogate, North Yorkshire from 23 to 25 May.

The Stent Summit (Ten Years of Stenting) will take place in London on 30 and 31 May, 1996. For further information, please contact: $\mathrm{Dr} U$ Sigwart, Department of Invasive Cardiology, Royal Brompton Hospital, Sydney Street, London SW3 6NP (tel: +44 1713518615 ; fax: +44 1713518614 ).
The European Lipoprotein Club will meet in Tutzing, near Munich, Germany on 11-14 September 1995. For participants associated with academic institutions there are no registration fees or charges for accommodation. Further information and abstract forms can be obtained from the secretary: Professor G Francheschini, c/o ELC 1995, Fondazione Giovanni Lorenzini, via Appiani 7, 20121 Milano, Italy (tel: +39 2 6471690; fax: +39 2 29007018). 